\title{
Decision Analysis: Omadacycline Relative to Moxifloxacin Among Hospitalized Community-Acquired Bacterial Pneumonia Patients at Risk of Clostridioides difficile Infection
}

\author{
Thomas P. Lodise ${ }^{1} \cdot$ Rohit Mistry $^{2} \cdot$ Kate Young $^{3} \cdot$ Kenneth LaPensee $^{4}$
}

Accepted: 23 January 2021

(c) The Author(s) 2021, corrected publication 2021

\begin{abstract}
Background and Objective Omadacycline is an aminomethylcycline antibiotic approved in the USA as once-daily intravenous/oral monotherapy for adults with community-acquired bacterial pneumonia (CABP). Omadacycline demonstrated noninferiority to the fluoroquinolone moxifloxacin in a phase III CABP trial; adverse-event rates were similar between treatment groups except for Clostridioides difficile infection (CDI), which occurred in $2 \%$ of moxifloxacin-treated patients and $0 \%$ of patients on omadacycline. Conceptual healthcare-decision analytic models were developed to better understand the economic implications of antibiotic selection and CDI risk in acute-care facilities.

Methods A conceptual healthcare-decision analytic model was created to estimate incremental costs associated with treating 100 hospitalized CABP patients with an initial 5-day inpatient regimen of omadacycline instead of moxifloxacin. The underlying model assumption was that treatment with omadacycline has the potential to reduce CDI events relative to moxifloxacin. The model included excess costs associated with each treatment group from admission through discharge. Attributable CDI cost per case in the moxifloxacin group varied from $\$ 15,000$ to $\$ 45,000$ (US\$). Omadacycline acquisition cost was \$300-600/day for 5 days.

Results At a CDI attributable cost per case of $\$ 30,000$ (base-case analyses), the incremental treatment cost (US\$) per 100 patients ranged from $\$ 300,000$ to $\$-120,000$ (cost savings). The excess CDI incidence in moxifloxacin-treated patients would need to be $5-10 \%$ for omadacycline to be cost-saving, assuming the attributable CDI cost is approximately $\$ 30,000$. Conclusion Targeted omadacycline use may reduce economic burden associated with hospitalized CABP patients treated with moxifloxacin if it can reduce excess cases of moxifloxacin-associated CDI.
\end{abstract}

\section{Introduction}

Clostridioides difficile infection (CDI), a common side effect of antibiotic therapy, is a substantial cause of morbidity and mortality across the USA. In 2011, there were nearly 500,000 cases of CDI and $~ 29,000$ CDI-associated deaths [1]. CDI also represents a major burden on the US healthcare system. The total costs attributable to CDI management are estimated to exceed $\$ 6.3$ billion annually in the

Thomas P. Lodise

Thomas.Lodise@acphs.edu

1 Albany College of Pharmacy and the Health Sciences, 106 New Scotland Avenue, Albany, NY 12189, USA

2 PAREXEL Access Consulting, London, UK

3 PAREXEL Access Consulting, Waltham, MA, USA

4 Paratek Pharmaceuticals, Inc., King of Prussia, PA, USA

\section{Key Points}

The CDC and Infectious Diseases Society of America encourages institutions to develop facility-specific antibiotic stewardship programs and consider restricting the use of antibiotics with highest risk for Clostridioides difficile infection (CDI), including fluoroquinolones and third-generation cephalosporins.

A conceptual healthcare-decision analytic model incorporating risks/costs of treatment-associated CDI was created to evaluate potential cost-saving opportunities of omadacycline versus the fluoroquinolone moxifloxacin among hospitalized patients with community-acquired bacterial pneumonia (CABP).

Omadacycline may reduce the economic burden of CABP if it can prevent five to ten excess cases of moxifloxacin-associated CDI per 100 treated patients. 
US [2]. Given the continuing high incidence and deleterious outcomes associated with CDI, prevention is a national priority, with a 2020 target to reduce CDI by $30 \%$ in the 2015 National Health Care-Associated Infections (HAI) Action Plan [3] CDI is also part of the hospital-acquired condition (HAC) reduction program, and hospitals have their Medicare payments reduced if their total HAC score is greater than the 75th percentile of all total HAC scores (i.e., the worstperforming quartile) [4]. Many US states mandate CDI data submission by hospitals as part of state HAI public reporting programs and CDI data are also collected at the federal level as part of the Centers for Medicare and Medicaid Services Hospital Inpatient Quality Reporting Program [4].

To aid in reducing the impact of CDI in acute-care facilities, the Centers for Disease Control and Prevention (CDC) provides data-driven strategies and interventions for addressing the burden of CDI in acute-care facilities [5]. In addition to infection control measures, the CDC encourages institutions to develop facility-specific antibiotic stewardship programs and consider restricting the use of antibiotics that have the highest risk for CDI, such as fluoroquinolones and third-generation cephalosporins [5, 6]. Similarly, the Infectious Diseases Society of America CDI guidelines strongly recommend restricting the use of broad-spectrum antibiotics, which include the aforementioned fluoroquinolones and cephalosporins [7]. These recommendations are based on the growing number of studies that have demonstrated hospitalonset CDI rates can be lowered by reducing the use of these agents [8-10].

Despite their increased propensity to cause CDIs, thirdgeneration cephalosporins and fluoroquinolones continue to be the preferred first-line agents for hospitalized patients with suspected or documented community-acquired bacteria pneumonia (CABP) [11]. One agent that may serve as a potential alternative antibiotic to these agents among patients with suspected or documented CABP is omadacycline (NUZYRA ${ }^{\circledR}$; Paratek Pharmaceuticals, Inc., King of Prussia, PA, USA). Omadacycline is an aminomethylcycline antibiotic approved in the USA as once-daily intravenous (IV) or oral monotherapy for the treatment of adults with CABP [12]. In the phase III Omadacycline for Pneumonia Treatment In the Community (OPTIC) Study (NCT02531438), omadacycline showed noninferiority to the fluoroquinolone moxifloxacin in the treatment of patients with CABP.

Omadacycline is derived from minocycline, a tetracycline antibiotic. Although all antibiotics have the potential to cause Clostridioides difficile-associated diarrhea (CDAD) [13], studies show that tetracycline antibiotics are associated with lower risk of CDI compared with fluoroquinolones [14, 15]. A growing body of preclinical and clinical evidence lends further credence to the notion that omadacycline may have a low propensity to induce CDI in the clinical setting relative to the fluoroquinolones. For example, in the OPTIC study, adverse-event rates were comparable between treatment groups except for CDI, which occurred in $8 / 388$ patients $(2 \%)$ who received moxifloxacin and 0/382 patients (0\%) who received omadacycline [12]. Furthermore, no cases of CDI were observed in the two phase III studies of omadacycline in patients with acute bacterial skin and skin-structure infections $[16,17]$. Results of a human colon model study also support the biologic plausibility of the differential CDI rates observed in OPTIC: omadacycline, in contrast to moxifloxacin, did not facilitate simulated CDI [18]. In addition to showing a low propensity to cause CDI, omadacycline demonstrated potent in vitro activity against $C$. difficile to comparators including doxycycline, metronidazole, and clindamycin [19], and demonstrated superior efficacy to vancomycin in a hamster model of CDAD [19].

To evaluate the potential economic value of omadacycline in the treatment of adults with suspected or confirmed CABP, a conceptual healthcare-decision analytic model was developed using a hospital perspective. This model sought to estimate the potential incremental costs associated with replacing moxifloxacin with omadacycline for hospitalized patients with suspected or documented CABP, taking the risk and cost of treatment-associated CDI into account. In this analysis, treatment of hospitalized CABP patients with omadacycline was assumed to have a lower propensity to induce CDI, relative to moxifloxacin. The goal of the study was to determine the number of excess moxifloxacin-associated CDI cases that needed to be avoided per 100 CABP cases with omadacycline to make incremental costs near zero (cost neutral) or to make omadacycline a cost-saving option from the hospital perspective.

\section{Methods}

\subsection{Model Description and Structure}

A deterministic framework from the US hospital perspective was used to develop the conceptual healthcare-decision analytic model that replaced the use of empiric intravenous moxifloxacin with omadacycline for the treatment of inpatients with suspected or documented CABP. The underlying model assumption was that treatment with omadacycline has a lower propensity to induce CDI relative to moxifloxacin and has the potential to avoid CDI events. The model assessed excess costs associated with each treatment group from patient admission to discharge. The costs associated with CDI post-discharge from the hospital perspective were also considered in the moxifloxacin arm of the model. Costs common to both treatments were not considered in the model. 
The population was 100 theoretical patients hospitalized for suspected or confirmed CABP (Fig. 1). Under the omadacycline scenario, all patients were assumed to be hospitalized for 5 days and were subsequently discharged from the hospital. Two different scenarios were considered for moxifloxacin. In the first, no patients experienced CDI with moxifloxacin and were discharged after 5 hospital days. In the second, a proportion of the 100 theoretical patients on moxifloxacin experienced CDI during or shortly after discharge, requiring a hospital readmission. If moxifloxacinassociated CDI occurred, it was assumed that hospitals would have incurred increased costs. The assumption for a 5-day hospital stay was derived from an analysis of hospitalized patients with CABP in the Vizient Health System Hospital Database [20]. The time period reflects the initial average inpatient stay (5 days) for either omadacycline or moxifloxacin in CABP.

\subsubsection{Model Inputs and Assumptions}

Only excess costs associated with each treatment were considered in the model. Emergency department and daily hospital costs were assumed to be equal during the initial 5-day treatment period, regardless of treatment assignment. CABP treatment duration for both groups was assumed to be 5 days. Moxifloxacin acquisition costs were assumed to be zero in the model to align with the acquisition cost of levofloxacin, the fluoroquinolone used most widely for hospitalized patients with CABP in the USA. Omadacycline acquisition costs (all reported here in US\$) varied between \$300/day and $\$ 600 /$ day in $\$ 150 /$ day increments. The current wholesale acquisition cost of omadacycline $100 \mathrm{mg}$ IV is $\$ 355.35$ per dose. With the loading Day 1 dose, the average wholesale acquisition cost (WAC) over 5 days is $\$ 426.42$ [21]. Rather than using this amount as the cost input for omadacycline, a range of costs was inputted for omadacycline, which was inclusive of the current daily omadacycline WAC, since the contracted price for a drug varies across hospitals and costs of a drug change over time. This also ensured readers would be able to best apply the findings of economic analysis based on the acquisition cost of omadacycline at their institution.

The excess moxifloxacin-associated CDI rate varied from 0 to $14 \%$, in $1 \%$ increments. This assumption was based on findings from the OPTIC trial [12]. In the OPTIC trial [12],

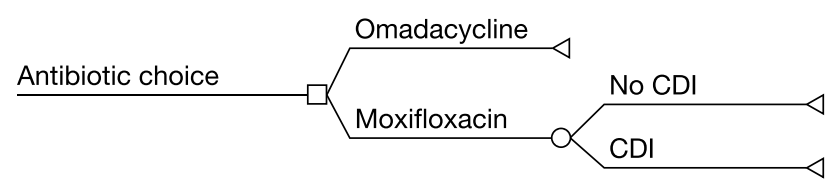

Fig. 1 Patient flow for empiric treatment of hospitalized adults with community-acquired bacterial pneumonia
CDI rates for each treatment group were stratified by the Davis risk score (DRS) [22], which is one of the most welldescribed predictive indices for 30-day risk of healthcareassociated CDI using readily available and clinically useful variables. Among patients in the phase 3 OPTIC study [12] with a DRS $\geq 6,14 \%$ of CABP patients treated with moxifloxacin developed CDI compared with no patients in the omadacycline-treated group, despite the balanced baseline CDI risk between treatment groups [23]. Two patients developed CDI after completion of treatment, whereas CDI occurred on Days 4-8 of treatment in the other six patients. This finding supports the CDI rates inputted in the model for the moxifloxacin-treated group.

The cost associated with a CDI in the model varied between $\$ 15,000$ and $\$ 45,000$, in $\$ 15,000$ increments. For the purposes of this analysis, $\$ 30,000$ was considered the base-case attributable cost associated with a CDI. Support for these inputs were derived from two systematic literature reviews and a recent CDI cost-of-illness study that indicated that the mean attributable costs associated with a healthcare-associated CDI are contained within this range [2, 24, 25]. In the meta-analysis by Zhang and colleagues [2], which combined 100-point estimates and ranges from 42 individual studies, hospital-onset CDI-attributable cost per case was estimated to be $\$ 34,157$ (90\% confidence interval (CI): \$33,134-\$35,180) [2]. In the systematic review of 45 cost-of-illness CDI studies between 1988 and June 2014, the mean attributable CDI costs (per patient/admission/episode/infection) ranged from $\$ 8,911$ to $\$ 30,049$ [24]. Most recently, Feuerstadt et al. reported that inpatient costs among CDI patients during the 12-month follow-up period ranged from $\$ 43,677$ for patients with no recurrences to $\$ 141,441$ for patients with at least three recurrences [25]. No additional adverse events were included in the model, as the incidence of other adverse events was found to be similar between omadacycline and moxifloxacin in the OPTIC study [12].

\subsubsection{Model Output and Analyses}

Incremental treatment cost was equal to the total cost for the scenario of 100 theoretical patients treated with omadacycline minus the total cost for the scenario of 100 theoretical patients treated with moxifloxacin. A positive incremental cost indicates increased cost with omadacycline, while a negative incremental cost suggests cost savings with omadacycline treatment compared to moxifloxacin treatment. Analyses were performed to determine the impact of varying the excess incidence of moxifloxacin-associated CDI (0-14\% in 1\% increments), cost of omadacycline (\$300-600/ day, in $\$ 150 /$ day increments for 5 days), and cost of CDI treatment $(\$ 15,000-45,000$, in $\$ 15,000$ increments) on the incremental treatment cost per 100 theoretical patients. A 
two-way sensitivity analysis was undertaken to determine the incremental treatment cost per 100 patients as a function of the CDI treatment cost per case. In the two-way sensitivity analysis, the cost of CDI treatment ranged between $\$ 0$ and $\$ 45,000$. The omadacycline IV daily acquisition cost was fixed at $\$ 426.42 /$ day for 5 days ( $\$ 2131$ total per treatment course) and the moxifloxacin daily acquisition cost was fixed at $\$ 0$.

\section{Results}

At a CDI-attributable cost per case of $\$ 30,000$ (basecase analyses), the incremental treatment cost per 100 patients ranged from $\$ 300,000$ to $\$-120,000$ (cost savings) (Table 1). At a CDI treatment cost per case of $\$ 30,000$, the excess incidence of CDI in moxifloxacin-treated patients would need to range between 5 and $10 \%$, depending on the acquisition cost of omadacycline (\$300-600/day), for omadacycline to be cost neutral. Analysis showed that for incremental cost per 100 patients to be near zero (cost neutral) at a CDI treatment cost per case of $\$ 15,000$, the excess incidence of CDI in moxifloxacin-treated patients would need to range between $10 \%$ and $20 \%$, depending on the acquisition cost of omadacycline (\$300-600/day). At the upper end of the CDI cost per case range $(\$ 45,000)$, negative incremental treatment costs were observed when the excess moxifloxacin-associated CDI incidence ranged between approximately 3 and 7\%, depending on the acquisition cost of omadacycline ( $\$ 300-600 /$ day).

Figure 2 shows results of the two-way sensitivity analysis, in which both cost of CDI per case and excess moxifloxacin-related CDI incidence were varied continuously and the omadacycline IV daily acquisition cost was fixed at at $\$ 426.42 /$ day for 5 days ( $\$ 2131$ total per treatment course). Omadacycline was cost minimizing at a moxifloxacinrelated excess CDI rate of approximately: (1) $14 \%$ at a mean CDI cost per case of $\$ 15,000$; (2) $7 \%$ at a CDI mean cost per case of $\$ 30,000$; and (3) $5 \%$ at a CDI mean cost per case of $\$ 45,000$.

\section{Discussion}

The incidence, mortality, and excess healthcare costs resulting from CDI in hospitalized patients in the USA are at historic highs [26]. C. difficile is one of three pathogens classified by the CDC as "an immediate public health threat that requires urgent and aggressive action" [27]. CDI prevention is a national priority and evidence-based criteria for curbing its spread are well described [7,28]. One of the major recommendations to reduce $\mathrm{CDI}$ is to restrict the use of fluoroquinolones and third-generation cephalosporins. While the benefits associated with the restriction of "high CDI risk"

Table 1 Incremental costs per 100 hospitalized CABP patients with omadacycline versus moxifloxacin

\begin{tabular}{|c|c|c|c|c|c|c|c|c|c|}
\hline \multirow{2}{*}{$\begin{array}{l}\text { CDI-attributable cost per case, US\$ } \\
\text { Omadacycline daily acquisition cost, } \\
\text { US\$ }\end{array}$} & \multicolumn{3}{|l|}{15,000} & \multicolumn{3}{|l|}{30,000} & \multicolumn{3}{|l|}{45,000} \\
\hline & 300 & 450 & 600 & 300 & 450 & 600 & 300 & 450 & 600 \\
\hline \multicolumn{10}{|l|}{$\begin{array}{l}\text { Excess CDI incidence with moxifloxa- } \\
\text { cin, } \%\end{array}$} \\
\hline 0 & 150,000 & 225,000 & 300,000 & 150,000 & 225,000 & 300,000 & 150,000 & 225,000 & 300,000 \\
\hline 1 & 135,000 & 210,000 & 285,000 & 120,000 & 195,000 & 270,000 & 105,000 & 180,000 & 255,000 \\
\hline 2 & 120,000 & 195,000 & 270,000 & 90,000 & 165,000 & 240,000 & 60,000 & 135,000 & 210,000 \\
\hline 3 & 105,000 & 180,000 & 255,000 & 60,000 & 135,000 & 210,000 & 15,000 & 90,000 & 165,000 \\
\hline 4 & 90,000 & 165,000 & 240,000 & 30,000 & 105,000 & 180,000 & $-30,000$ & 45,000 & 120,000 \\
\hline 5 & 75,000 & 150,000 & 225,000 & 0 & 75,000 & 150,000 & $-75,000$ & 0 & 75,000 \\
\hline 6 & 60,000 & 135,000 & 210,000 & $-30,000$ & 45,000 & 120,000 & $-120,000$ & $-45,000$ & 30,000 \\
\hline 7 & 45,000 & 120,000 & 195,000 & $-60,000$ & 15,000 & 90,000 & $-165,000$ & $-90,000$ & $-15,000$ \\
\hline 8 & 30,000 & 105,000 & 180,000 & $-90,000$ & $-15,000$ & 60,000 & $-210,000$ & $-135,000$ & $-60,000$ \\
\hline 9 & 15,000 & 90,000 & 165,000 & $-120,000$ & $-45,000$ & 30,000 & $-255,000$ & $-180,000$ & $-105,000$ \\
\hline 10 & 0 & 75,000 & 150,000 & $-150,000$ & $-75,000$ & 0 & $-300,000$ & $-225,000$ & $-150,000$ \\
\hline 11 & $-15,000$ & 60,000 & 135,000 & $-180,000$ & $-105,000$ & $-30,000$ & $-345,000$ & $-270,000$ & $-195,000$ \\
\hline 12 & $-30,000$ & 45,000 & 120,000 & $-210,000$ & $-135,000$ & $-60,000$ & $-390,000$ & $-315,000$ & $-240,000$ \\
\hline 13 & $-45,000$ & 30,000 & 105,000 & $-240,000$ & $-165,000$ & $-90,000$ & $-435,000$ & $-360,000$ & $-285,000$ \\
\hline 14 & $-60,000$ & 15,000 & 90,000 & $-270,000$ & $-195,000$ & $-120,000$ & $-480,000$ & $-405,000$ & $-330,000$ \\
\hline
\end{tabular}

Cost of hospital-onset Clostridioides difficile infection (CDI) tested over the range of values reported in the literature [2, 24, 25]. Negative \$ values indicate costs savings with omadacycline in the model, at the given omadacycline cost and CDI incidence rate 
antibiotics have been clearly demonstrated [29], it can be challenging for many healthcare facilities to fully adopt this recommendation. For example, dedicated antibiotic stewardship programs are absent from many healthcare facilities, limiting the ability to curtail the use of these high CDI risk antibiotics. In addition, fluoroquinolones and third-generation cephalosporins are the cornerstones of treatment in several expert guidelines, making it difficult to limit their use. Furthermore, only a limited number of alternative antibiotics are available to the fluoroquinolone and third-generation cephalosporins for several common bacterial infections in hospitalized patients [11, 30, 31]. Most notably, the 2019 guidelines for community-acquired pneumonia continue to recommend third-generation cephalosporin-based regimens as first-line therapies for hospitalized CABP patients [11].

Cognizant of the urgent national concerns with CDI, a conceptual healthcare-decision analytic model was developed to better understand the economic implications of the potentially lower CDI risk associated with omadacycline relative to fluoroquinolones. Rather than examining incremental cost-effectiveness ratios (or patient-centered outcomes such as quality-adjusted life years), a deterministic model was created to assess the value associated with omadacycline relative to moxifloxacin by quantifying the number of excess cases of moxifloxacin-associated CDI that needed to be avoided per 100 patients in order for omadacycline to be cost neutral for the hospital. While it is well established that the primary goals of antibiotic stewardship are to optimize patient outcomes and avoid unintended consequences

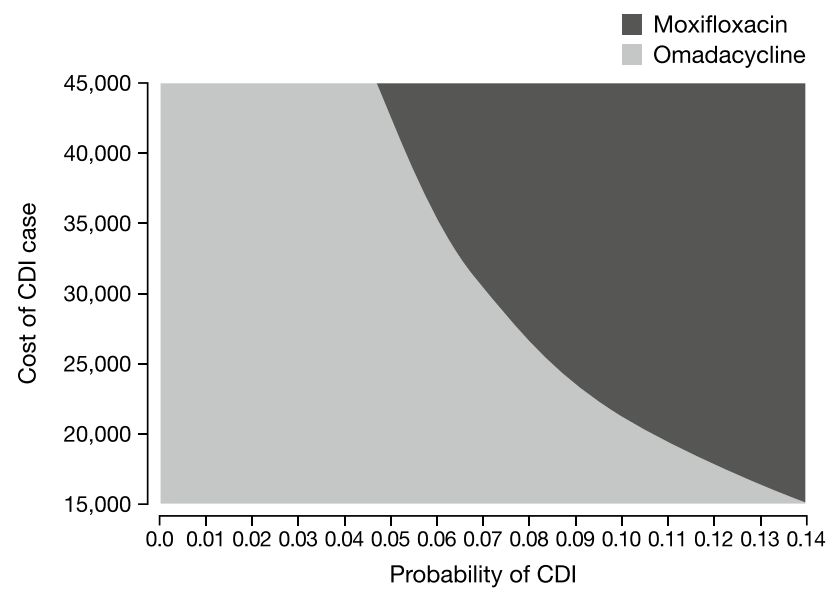

Fig. 2 Two-way sensitivity analysis of the incremental cost (\$) per 100 hospitalized CABP patients with omadacycline relative to moxifloxacin. The omadacycline intravenous daily acquisition cost was fixed at $\$ 426.24$ for 5 days $(\$ 2,131.20$ per treatment course), and the moxifloxacin daily acquisition cost was fixed at $\$ 0$. CABP community-acquired bacterial pneumonia, CDI Clostridioides difficile infection. Excess moxifloxacin-related CDI incidence per 100 patients varied between 0 and $14 \%$, and CDI mean cost per case ranged from $\$ 15,000$ to $\$ 45,000$ of antibiotic use like CDI, containment of overall and antibiotic-related costs are also significant goals [32]. Thus, we believe it was important to examine an endpoint in the analysis that captured all the critical metrics of antibiotic stewardship: patient outcomes, CDI prevention, and cost of care. Our focus on these aspects also aligned with the tenets of value-based healthcare, which place emphasis on quality and efficiency of care.

Overall, results from the analyses indicated that omadacycline use has the potential to reduce the healthcare economic burden associated with CDI in patients hospitalized for CABP who are currently treated with moxifloxacin if it can prevent $\sim 5-10$ cases of moxifloxacin-associated CDI per 100 treated patients, assuming the attributable CDI cost is approximately $\$ 30,000$. A number of studies have been performed to determine the mean attributable costs associated with a healthcare-associated CDI and it is estimated to be approximately $\$ 15,000-45,000[2,24,25]$. At the upper end of this range, the number of moxifloxacin-associated CDI cases that needed to be prevented for omadacycline to be cost neutral was $\sim 3-7$ per 100 patients. At the lower end, the number of moxifloxacin-associated CDI cases that needed to be prevented for omadacycline to be cost neutral was 10-20 per 100 patients. Given the morbidity, mortality, healthcare expenditures, and potential Medicare reimbursement reductions associated with CDI, hospitals and clinicians should consider the incremental cost findings in relation to overall benefits of potentially preventing a CDI case from the patient, hospital, and societal perspectives. In addition, it may be that omadacycline use in hospitalized suspected or documented CABP, as a quality control measure to potentially reduce the occurrence of fluoroquinolone-associated CDI, is best suited for patient populations at greatest risk for CDI. Such populations are well described in the literature and include patients with multiple comorbidities, prior CDI, extensive prior receipt of "high CDI risk" antibiotics, and receipt of proton pump inhibitors [22]. This proposed recommendation for use in high CDI risk patients is consistent with the results of the OPTIC trial [12], in which $14 \%$ of moxifloxacin-treated patients with a DRS $\geq 6$ developed CDI compared with no patients in the omadacycline-treated group [23].

Several points should be noted when interpreting the findings of the present analysis. First, we examined the potential incremental costs associated with replacing moxifloxacin with omadacycline for hospitalized patients with suspected or documented CABP. Our findings are not unique to omadacycline and could be applied to any antibiotic that confers a lower risk of CDI relative to fluoroquinolones. Second, we focused on excess rather than total costs because many costs (hospitalization for initial treatment period, nursing time) would be the same regardless of the choice of antimicrobial agent. Third, we did not factor in a series of outcomes 
and costs such as patient satisfaction/quality of life/productivity, due to lack of comparator data on these endpoints between omadacycline and moxifloxacin. Fourth, we did not incorporate mortality - which occurs in a fair proportion of patients with CDI-into the model $[1,2,33]$. The conceptual models assumed that omadacycline did not lead to any cases of CDI. Thus, the "cost neutral" moxifloxacinassociated CDI incidence thresholds identified in the analyses reflect the excess CDI cases with moxifloxacin relative to omadacycline, rather than overall absolute numbers of moxifloxacin-associated CDI cases. Finally, this study only evaluated incremental costs associated with averting moxifloxacin-associated CDI. The incremental costs presented in this study should be offset with the frequencies and costs of other potential toxicities that could occur with both omadacycline and moxifloxacin.

\section{Conclusions}

An important clinical, economic, and public health consideration with the use of fluoroquinolones is the occurrence of CDI. Findings from the model presented here illustrate the potential economic impact associated with reductions in CDI rates following the use of antibiotics that have low CDI risk, such as omadacycline, compared with fluoroquinolones, when treating patients hospitalized for CABP. Based on the model's assumptions and inputs, this analysis suggests that omadacycline use has the potential to reduce the economic burden associated with moxifloxacin use in hospitalized CABP patients, if it can prevent $~ 5-10$ cases of moxifloxacin-associated CDI per 100 treated patients, assuming the attributable CDI cost is approximately $\$ 30,000$. Hospitals aspiring to prevent CDI, improve patient outcomes, and contain costs by avoidance of fluoroquinolone-associated CDI should examine their hospital-specific rates of CDI to determine whether the replacement of fluoroquinolones with an agent such as omadacycline can reduce healthcare costs and prevent CDI among patients at high CDI risk [7, 22]. Like all studies of this nature, the findings in this analysis require validation in real-world settings. Future studies should explore the incidence, burden, and clinical impact of fluoroquinolone-associated CDI in CABP patients.

Acknowledgements The authors thank the PAREXEL Access RWE team, who provided real-world data used in this study. Medical editorial assistance, funded by Paratek Pharmaceuticals, Inc., was provided by Holly Tomlin, MPH, CMPP for Innovative Strategic Communications.

\section{Declaration}

Funding This work was supported by Paratek Pharmaceuticals, Inc.
Conflict of interest KLP is a former employee of Paratek Pharmaceuticals, Inc. TL and KLP have been employed as consultants by Paratek Pharmaceuticals, Inc. RM and KY are former employees of PAREXEL, which provided consulting services to Paratek Pharmaceuticals, Inc.

Ethics approval Not applicable.

Consent to participate Not applicable.

Consent for publication Not applicable.

Data availability Paratek Pharmaceuticals has a commitment to ensure that access to clinical trial data is available to regulators, researchers, and trial participants, when permitted, feasible and appropriate. Requests for de-identified patient-level data may be submitted to medinfo@paratekpharma.com for review.

Code availability Not applicable.

Author contributions TPL generated the study concept; was involved in developing the model structure, inputs, and outputs; and participated in manuscript writing, preparation, and publication. RM and KY developed the analysis, interpreted the data, and revised and reviewed the manuscript. KLP developed the decision models and performed the decision analysis; and participated in manuscript writing, preparation, and publication. All authors approved the final version.

Open Access This article is licensed under a Creative Commons Attribution-NonCommercial 4.0 International License, which permits any non-commercial use, sharing, adaptation, distribution and reproduction in any medium or format, as long as you give appropriate credit to the original author(s) and the source, provide a link to the Creative Commons licence, and indicate if changes were made. The images or other third party material in this article are included in the article's Creative Commons licence, unless indicated otherwise in a credit line to the material. If material is not included in the article's Creative Commons licence and your intended use is not permitted by statutory regulation or exceeds the permitted use, you will need to obtain permission directly from the copyright holder. To view a copy of this licence, visit http://creativecommons.org/licenses/by-nc/4.0/.

\section{References}

1. Lessa FC, Mu Y, Bamberg WM, Beldavs ZG, Dumyati GK, Dunn $\mathrm{JR}$, et al. Burden of Clostridium difficile infection in the United States. N Engl J Med. 2015;372(9):825-34.

2. Zhang S, Palazuelos-Munoz S, Balsells EM, Nair H, Chit A, Kyaw $\mathrm{MH}$. Cost of hospital management of Clostridium difficile infection in United States: a meta-analysis and modelling study. BMC Infect Dis. 2016;16(1):447.

3. National Action Plan to Prevent Health Care-Associated Infections: road map to elimination. http://www.apic.org/Resource_/ TinyMceFileManager/Advocacy-PDFs/Tab_B_Phase_IV_Draft_ with_edits_v3.0.pdf. Accessed 24 Mar 2020.

4. Centers for Medicare \& Medicaid Services. Hospital-Acquired Condition Reduction Program (HACRP). https://www.cms.gov/ medicare/medicare-fee-for-service-payment/acuteinpatientpps/ hac-reduction-program.html. Accessed 24 Mar 2020.

5. Centers for Disease Control and Prevention. Strategies to prevent Clostridioides difficile infection in acute care facilities. 
https://www.cdc.gov/hai/prevent/cdi-prevention-strategies.html. Accessed 24 Mar 2020.

6. Centers for Disease Control and Prevention. Core elements of hospital antibiotic stewardship programs. https://www.cdc.gov/ antibiotic-use/healthcare/implementation/core-elements.html\#_ ENREF_15. Accessed 24 Mar 2020.

7. McDonald LC, Gerding DN, Johnson S, Bakken JS, Carroll KC, Coffin SE, et al. Clinical Practice Guidelines for Clostridium difficile Infection in Adults and Children: 2017 update by the Infectious Diseases Society of America (IDSA) and Society for Healthcare Epidemiology of America (SHEA). Clin Infect Dis. 2018;66(7):e1-48.

8. Shea KM, Hobbs ALV, Jaso TC, et al. Effect of a health care system respiratory fluoroquinolone restriction program to alter utilization and impact rates of Clostridium difficile infection. Antimicrob Agents Chemother. 2017;61(6):e00125-e217. https://doi. org/10.1128/AAC.00125-17.

9. Dingle KE, Didelot X, Quan TP, Eyre DW, Stoesser N, Golubchik T, et al. Effects of control interventions on Clostridium difficile infection in England: an observational study. Lancet Infect Dis. 2017;17(4):411-21.

10. Kazakova SV, Baggs J, McDonald LC, Yi SH, Hatfield KM, Guh A, et al. Association between antibiotic use and hospital-onset Clostridioides difficile infection in U.S. acute care hospitals, 2006-2012: an ecologic analysis. Clin Infect Dis. 2020;70(1):11-8.

11. Metlay JP, Waterer GW, Long AC, Anzueto A, Brozek J, Crothers K, et al. Diagnosis and treatment of adults with communityacquired pneumonia. An official clinical practice guideline of the American Thoracic Society and Infectious Diseases Society of America. Am J Respir Crit Care Med. 2019;200(7):e45-67.

12. Stets R, Popescu M, Gonong JR, Mitha I, Nseir W, Madej A, et al. Omadacycline for community-acquired bacterial pneumonia. $\mathrm{N}$ Engl J Med. 2019;380(6):517-27.

13. NUZYRA ${ }^{\mathrm{TM}}$ package insert. https://www.accessdata.fda.gov/ drugsatfda_docs/label/2018/209816_2098171bl.pdf. Accessed 5 Mar 2020.

14. Tariq R, Cho J, Kapoor S, Orenstein R, Singh S, Pardi DS, et al. Low risk of primary Clostridium difficile infection with tetracyclines: a systematic review and metaanalysis. Clin Infect Dis. 2018;66(4):514-22.

15. Deshpande A, Pasupuleti V, Thota P, Pant C, Rolston DDK, Sferra TJ, et al. Community-associated Clostridium difficile infection and antibiotics: a meta-analysis. J Antimicrob Chemother. 2013;68(9):1951-61.

16. O'Riordan W, Green S, Overcash JS, Puljiz I, Metallidis S, Gardovskis $J$, et al. Omadacycline for acute bacterial skin and skinstructure infections. N Engl J Med. 2019;380(6):528-38.

17. O'Riordan W, Cardenas C, Shin E, Sirbu A, Garrity-Ryan L, Das $\mathrm{AF}$, et al. OASIS-2 Investigators. Once-daily oral omadacycline versus twice-daily oral linezolid for acute bacterial skin and skin structure infections (OASIS-2): a phase 3, double-blind, multicentre, randomised, controlled, non-inferiority trial. Lancet Infect Dis. 2019;19(10):1080-90.

18. Moura IB, Buckley AM, Ewin D, Shearman S, Clark E, Wilcox $\mathrm{MH}$, et al. Omadacycline gut microbiome exposure does not induce Clostridium difficile proliferation or toxin production in a model that simulates the proximal, medial, and distal human colon. Antimicrob Agents Chemother. 2019;63(2):e01581-e1618. https://doi.org/10.1128/AAC.01581-18.
19. Kim O, Leahy RG, Traczewski M et al. Activity and efficacy of omadacycline against Clostridium difficile. Presented at ECCMID 2016, Poster P1325.

20. LaPensee K, Mistry R, Lodise T. Budget impact model of omadacycline on replacing a proportion of existing treatment options among patients who present to the emergency department with acute bacterial skin and skin structure infections. Am Health Drug Benefits. 2019;12(1-Supplement 2):S13-S24.

21. RED BOOK (Micromedex $\left.{ }^{\circledR}\right)$. Wholesale acquisition cost of intravenous omadaycycline. Accessed 18 Nov 2020.

22. Davis ML, Sparrow HG, Ikwuagwu JO, Musick WL, Garey KW, Perez KK. Multicentre derivation and validation of a simple predictive index for healthcare-associated Clostridium difficile infection. Clin Microbiol Infect. 2018;24(11):1190-4.

23. Rodriguez M, Wright K, Noble R. Predicted risk and observed occurrence of Clostridioides difficile infection in patients with community-acquired bacterial pneumonia treated with omadacycline or moxifloxacin. Presented at ECCMID 2020, Poster P4548.

24. Nanwa N, Kendzerska T, Krahn M, Kwong JC, Daneman N, Witteman W, et al. The economic impact of Clostridium difficile infection: a systematic review. Am J Gastroenterol. 2015;110(4):511-9.

25. Feuerstadt P, Strong L, Dahdal DN, Sacks N, Lang K, Nelson WW. Healthcare resource utilization and direct medical costs associated with index and recurrent Clostridioides difficile infection: a real-world data analysis. J Med Econ. 2020;23(6):603-9.

26. Vindigni SM, Surawicz CMC. difficile infection: changing epidemiology and management paradigms. Clin Transl Gastroenterol. 2015;6(7):e99.

27. 28Centers for Disease Control and Prevention. Antibiotic resistance threats in the United States, 2013. https://www.cdc.gov/drugr esistance/pdf/ar-threats-2013-508.pdf. Accessed 28 Aug 2019.

28. Office of Disease Prevention and Health Promotion. National targets and metrics. https://health.gov/hcq/prevent-hai-measures.asp. Accessed 28 Aug 2019.

29. Guh A, Mu Y, Winston LG, Johnston H, Olson D, Farley MM, et al. Trends in U.S. burden of Clostridioides difficile infection and outcomes. N Engl J Med. 2020;382(14):1320-30.

30. Solomkin JS, Mazuski JE, Bradley JS, Rodvold KA, Goldstein EJC, Baron EJ, et al. Diagnosis and management of complicated intra-abdominal infection in adults and children: Guidelines by the Surgical Infection Society and the Infectious Diseases Society of America. Clin Infect Dis. 2010;50(2):133-64.

31. Kalil AC, Metersky ML, Klompas M, Muscedere J, Sweeney DA, Palmer LB, et al. Management of adults with hospital-acquired and ventilator-associated pneumonia: 2016 Clinical Practice Guidelines by the Infectious Diseases Society of America and the American Thoracic Society. Clin Infect Dis. 2016;63(5):e61-111.

32. Barlam TF, Cosgrove SE, Abbo LM, MacDougall C, Schuetz AN, Septimus EJ, et al. Implementing an antibiotic stewardship program: Guidelines by the Infectious Diseases Society of America and the Society for Healthcare Epidemiology of America. Clin Infect Dis. 2016;62(10):e51-77.

33. Chalmers JD, Akram AR, Singanayagam A, Wilcox MH, Hill AT. Risk factors for Clostridium difficile infection in hospitalized patients with community-acquired pneumonia. J Infect. 2016;73(1):45-53. 